\title{
Testosterone and dihydrotestosterone differentially improve cognition in aged female mice
}

\author{
Ted S. Benice ${ }^{1}$ and Jacob Raber ${ }^{1,2,3,4}$ \\ ${ }^{1}$ Department of Behavioral Neuroscience, Oregon Health and Science University, Portland, Oregon 97239, USA; ${ }^{2}$ Department of \\ Neurology, Oregon Health and Science University, Portland, Oregon 97239, USA; ${ }^{3}$ Division of Neuroscience ONPRC, Oregon \\ Health and Science University, Portland, Oregon 97239, USA
}

\begin{abstract}
Compared with age-matched male mice, female mice experience a more severe age-related cognitive decline (ACD). Since androgens are less abundant in aged female mice compared with aged male mice, androgen supplementation may enhance cognition in aged female mice. To test this, we assessed behavioral performance on a variety of tasks in 22- to 24-mo-old gonadally intact female mice treated for 6 wk with silastic capsules containing either testosterone (T) or dihydrotestosterone (DHT) or empty capsules (placebo). Compared with placebo-treated mice, spatial memory retention in the water maze was enhanced by testosterone treatment, but not DHT treatment. In contrast, DHT treatment improved passive avoidance (PA) retention, while T treatment only did so marginally. These data support that androgen supplementation in old female mice improves cognitive performance differentially depending upon the type of hormone treatment and cognitive task.
\end{abstract}

Age-related cognitive decline (ACD) refers to a generalized disruption of cognitive function that occurs during the aging process in the absence of overt neurodegenerative disease (Small et al. 1995; Tisserand and Jolles 2003). ACD is associated with declines in multiple cognitive domains, including spatial cognition (Driscoll et al. 2005), verbal memory (Small et al. 1995), attention (Tisserand and Jolles 2003), and executive function (Hanninen et al. 1997). Along with functional cognitive decline, there are associated agerelated disruptions in cortical and hippocampal volumes (Driscoll et al. 2003), volumes of cortical gray matter and cerebrospinal fluid (Smith et al. 2007), and white matter integrity (Silbert et al. 2008). As the population ages, it becomes increasingly important to find ways to prevent and treat ACD.

In rodents, there is a clear sex difference in age of ACD onset (Markowska 1999; Frick et al. 2000; Benice et al. 2006), as assessed by some, but not all, cognitive tasks, with females showing earlier onset compared with males. However, the cognitive endpoint with advanced age is similar between the sexes. Sex differences in the onset of age-related cognitive performance deficits may be partly due to differences in gonadal hormone levels. Female rats experience earlier declines in hippocampus-dependent spatial memory performance compared with male rats (Markowska 1999). In addition, age-related performance deficits on a spatial "working memory" task in aged female rats are exacerbated by estrogen withdrawal and recovered by chronic systemic replacement with estradiol combined with priming subcutaneous injections with estradiol (Markowska and Savonenko 2002). Middle-aged female mice show greater age-related impairments in the spatial "reference"-memory water maze task compared with middle-aged males (Frick et al. 2000; Benice et al. 2006). Similar to rats, these deficits are antagonized by daily subcutaneous injections of estradiol (Frick et al. 2002). In mice that express human apolipoprotein E4, a major risk factor for sporadic Alzheimer's disease, females show greater impairments than males in spatial reference memory performance (Raber et al. 2000) that are improved by $8 \mathrm{~d}$ of systemic treatment with either testosterone (T) or dihydrotestoster-

\footnotetext{
${ }^{4}$ Corresponding author.
}

E-mail raberj@ohsu.edu; fax (503) 494-6877.

Article is online at http://www.learnmem.org/cgi/doi/10.1101/lm.1428209. one (DHT) capsules (Raber et al. 2002). Although also affected by hippocampal lesions (Stubley-Weatherly et al. 1996), the passive avoidance (PA) task in rodents is very sensitive to amygdala lesions (Swartzwelder 1981). Compared with male mice, female mice also show greater age-related declines in PA performance (Benice et al. 2006), and PA performance in aged female mice can be improved by giving subcutaneous injections of estradiol after training (Frye et al. 2005). In the novel object recognition memory task, which is hippocampus dependent only with long 24-h delays between learning and retrieval (Hammond et al. 2004), performance is also disrupted by age in female mice (Vaucher et al. 2002). Although removal of estradiol by ovariectomy further impairs performance (Vaucher et al. 2002), supplementation with post-training intraperitoneal injections of estradiol may not recover performance on this task in aged females (Gresack et al. 2007). Though the effects of estrogens on cognitive performance in aged females have been studied extensively, no studies have specifically investigated whether androgen supplementation affects spatial memory in the water maze navigational task, emotional memory as assessed in the PA task, or object recognition memory performance in aged female rodents. In addition, since males have higher levels of circulating androgens compared with females at all ages, it is possible that androgens may confer some protection against ACD.

Androgens are steroid compounds that bind to and activate the nuclear androgen receptor (AR), which affects neuronal function by altering gene transcription (McPhaul and Young 2001). ARs are expressed at high levels in brain areas important for cognition such as the hippocampus, amygdala, and cerebral cortex in rodents (Simerly et al. 1990), and the levels of AR in the brain are modulated by androgens (Lu et al. 1998). Importantly, the morphology of neurons in these regions is sensitive to manipulations in androgen levels. For example, the density of dendritic spine synapses in the CA1 region of the hippocampus is increased by DHT treatment in ovariectomized female rats (Leranth et al. 2004), and androgen administration to neonatal female rats increases the adult hippocampal dentate gyrus volume (Isgor and Sengelaub 1998). In addition, neuronal and regional volumes in the amygdala are increased by $\mathrm{T}$ administration both in neonatal (Cooke 2006) and adult female rats (Morris et al. 2008). 
To characterize the effects of androgen supplementation on cognitive performance in aged female mice, we treated 22- to 24mo-old female mice with T, nonaromatizable DHT, or placebo and tested performance on spatial memory in the water maze, object recognition memory, and PA tasks. It is important to note that $\mathrm{T}$ can be readily aromatized to estradiol in the brain and periphery. Thus, effects of $\mathrm{T}$ on cognitive performance cannot be interpreted as wholly androgen effects. However, the potent androgen dihydrotestosterone cannot be aromatized and its effects are therefore androgenic. Thus, we hypothesized that if androgenic mechanisms, apart from aromatization to estradiol, are beneficial for cognitive task performance in aged female mice, then DHT supplementation as well as $\mathrm{T}$ supplementation would improve performance in all three of the cognitive tasks. However, if cognitive performance enhancement relies upon aromatization to estradiol, then DHT supplementation would not improve performance. We also measured anxiety-like behavior and motor coordination as these behaviors can potentially contribute to differences in learning and memory (Benice et al. 2006).

\section{Results}

\section{Condition of the aged mice during the procedures}

All mice survived the capsule implantation procedure, and none showed obvious signs of ill effects due to surgery. One T-treated mouse died in the days between being tested in the referencememory Morris water maze and roto-rod. All other mice survived the testing regimen, were ambulatory and active, had no debilitating cases of hair loss and dermatitis, and had no obvious loss of sight.

\section{Hormone levels}

Both T $\left(F_{(2,16)}=572, P<0.001\right)$ and DHT $\left(F_{(2,16)}=77.1, P<0.001\right)$ supplementation successfully elevated hormone levels over placebo (Fig. 1). T levels were significantly higher in the mice supplemented with T $(n=6)$ compared with either placebo $(n=5)$ or DHT-treated mice ( $n=6 ; P<0.05$ by Duncan's post-hoc test). Similarly, DHT levels were highest in the mice supplemented with T compared with either DHT- or placebo-treated mice $(P<0.05$ by Duncan's post-hoc test). DHT levels were higher in the DHTtreated mice compared with placebo-treated mice $(P<0.05$ by Duncan's post-hoc test), but T levels were not.

\section{Open field}

Treatment with T $(n=11)$, DHT $(n=10)$, or placebo $(n=8)$ did not affect measures of anxiety or activity in the open field (Fig. 2). There was no effect of hormone treatment on percentage time spent in the center of the open field $\left(F_{(2,28)}=1.18, P=0.32\right.$; Fig. $2 \mathrm{~A})$. There was also no effect of hormone treatment on total distance moved in the open field $\left(F_{(2,28)}=1.81, P=0.18\right.$; Fig. $\left.2 \mathrm{~B}\right)$.

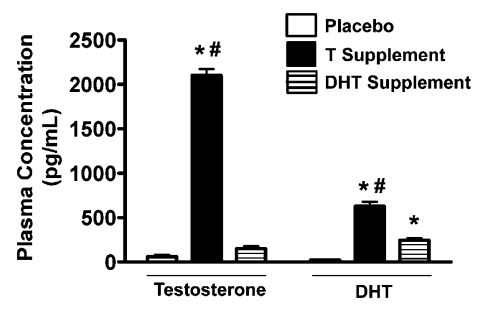

Figure 1. Plasma $T$ and $D H T$ levels for mice supplemented with either $T$ $(n=6)$ or $\mathrm{DHT}(n=6)$ or given placebo $\left(n=5\right.$; empty capsules). ${ }^{*} P<0.05$ vs. placebo treated group by Duncan's post-hoc test following ANOVA. ${ }^{\#} P<0.05$ vs. DHT-treated group by Duncan's post-hoc test following ANOVA.

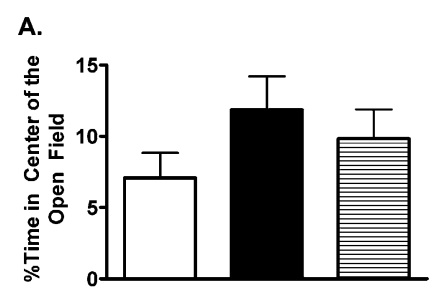

B.

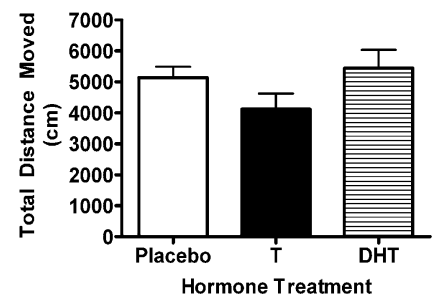

Figure 2. Measures of anxiety $(A)$ and activity $(B)$ in the open field. Neither T nor DHT supplementation significantly changed anxiety or activity.

\section{Novel object $(\mathrm{NO})$ and novel location $(\mathrm{NL})$ memory}

Treatment with T $(n=11)$ or DHT $(n=10)$ did not improve object recognition compared with treatment with placebo $(n=8$; Fig. 3$)$. There was no effect of hormone treatment on either the novel object recognition score $\left(F_{(2,28)}=0.886, P=0.43\right.$; Fig. $\left.3 \mathrm{~A}\right)$ or the novel location recognition score $\left(F_{(2,28)}=0.68, P=0.51\right.$; Fig. 3B $)$. In addition, there was also no difference between the treatment groups in total time spent exploring the objects over the five $\mathrm{NO} /$ NL trials $\left(F_{(2,26)}=0.43, P=0.66\right.$; Fig. 3C) and no interaction between treatment group and $\mathrm{NO} / \mathrm{NL}$ trials for this measure $\left(F_{(8,104)}=1.2, P=0.29\right)$.

\section{Reference-memory Morris water maze}

Performance during the training phase of the water maze task was unaffected by treatments with T $(n=11)$, DHT $(n=10)$, or placebo $(n=8$; Fig. 4B,C). Swim speeds during the visible training sessions did not differ between the treatment groups $\left(F_{(2,28)}=2.42, P=\right.$ 0.11; Fig. 4A). However, there was a trend toward a hormone treatment effect on swim speed during the hidden training sessions $\left(F_{(2,28)}=2.69, P=0.09\right)$. There was an effect of session on escape latency in both the visible $\left(F_{(3,75)}=3.95, P=0.01\right)$ and hidden sessions $\left(F_{(5,130)}=7.87, P<0.001\right)$, indicating that the mice improved performance during training (Fig. 4B). The same was found for total distance moved (Fig. $4 \mathrm{C})$ in both the visible $\left(F_{(3,75)}=8.42\right.$, $P<0.01)$ and hidden $\left(F_{(5,130)}=13.1, P<0.001\right)$ sessions. However, there was no effect of hormone treatment on escape latency (or total distance moved) in either visible $\left(F_{(2,26)}=0.75, P=0.48\right)$ or hidden $\left(F_{(2,26)}=1.23, P=0.31\right)$ trials, and there were no significant interactions between session and hormone treatment.

In contrast to the training performance, T-treated mice showed improved performance during the probe trial memory test after the last day of training (Fig. 4F). The T-treated females showed spatial bias for the target quadrant indicating spatial learning of the target location after day 3 of hidden training $(P<0.05$ by a priori planned contrast), but not after day 1 (Fig. 4D) or day 2 (Fig. 4E). In contrast, neither placebo- or DHT-treated females ever showed spatial bias for the target quadrant.

\section{Roto-rod}

There was a significant effect of training trial on roto-rod performance $\left(F_{(8,200)}=3.87, P<0.001\right)$ indicating improvement in performance over the nine training trials (Fig. 5). However, there 

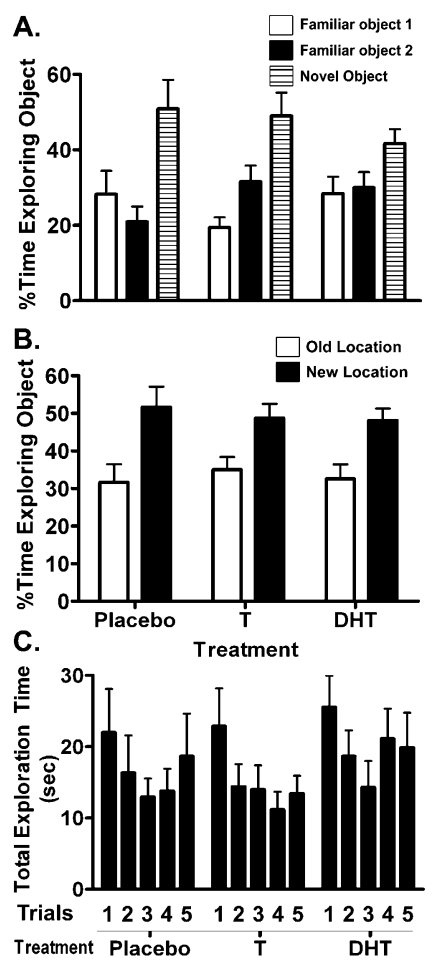

Figure 3. Novel object $(A)$, novel location $(B)$ recognition performance, and total exploration time during the five trials of the NO/NL task (C). Preference for the novel object during trial 5 (novel object test), and for a familiar object in a novel location vs. the old location, were unaffected by T or DHT supplementation, as were total exploration time during the five trials of the NO/NL task.

was no effect of treatment with T $(n=10)$, DHT $(n=11)$, or placebo $\left(n=8 ; F_{(2,25)}=1.71, P=0.30\right)$, and there was no interaction between hormone treatment and training trial.

\section{Passive avoidance}

DHT $(n=10)$ treatment, but not T treatment $(n=10)$, significantly increased latency to enter the dark chamber in the PA memory task compared with placebo ( $n=8$; Fig. 6$)$. There was no hormone treatment effect on test acquisition $\left(\chi^{2}=2.07, P=0.35\right.$; Fig. 6A). However, there was an effect of hormone treatment on PA memory retention $24 \mathrm{~h}$ after training $\left(\chi^{2}=6.87, P=0.03\right.$; Fig. $6 \mathrm{~B})$. Compared with placebo, DHT significantly increased latency to enter the dark compartment during the memory retention test trial $(Z=2.54, P=0.02)$, but $\mathrm{T} \operatorname{did} \operatorname{not}(Z=1.67, P=0.12)$. However, there was no significant difference in PA memory performance between T- and DHT-treated females $(Z=0.93, P=0.48)$. For the placebo-treated mice, six out of eight mice entered the dark compartment during the test trial. In contrast, only one out of 10 DHT-treated mice entered the dark compartment. The performance of T-treated mice was in between placebo- and DHT-treated mice, with four out of 10 mice entering the dark compartment. Similar to latency to enter, $\chi^{2}$ tests revealed that a smaller proportion of DHT-treated mice entered the dark compartment compared with placebo $\left(\chi^{2}=7.9, P<0.05\right)$, but there were no significant differences between either $\mathrm{T}$ or placebo or between $\mathrm{T}$ and DHT.

\section{Discussion}

There were three major findings from this study in 22- to 24-moold female mice: (1) Compared with placebo-treatment, supple- mentation with T, but not DHT, we found improved spatial reference memory in the Morris water maze; (2) compared with placebo treatment, supplementation with DHT improved PA memory, whereas T only marginally improved performance; and (3) hormone supplementation had no effect on either location or object novelty recognition memory open field measures of anxiety, or roto-rod performance. These data are the first to explore the cognitive-enhancing effects of androgen hormone supplementation in aged female rodents. The data support the hypothesis that androgen hormone supplementation is beneficial for some aspects of cognitive function in aged female mice. Also, androgenic mechanisms may present different targets for treatment of separate aspects of ACD.

The aged female mice treated with placebo in this study failed to show any target quadrant preference during any probe trial. This is similar to previous findings using the same strain of female mice aged 18- to 20-mo old (Benice et al. 2006). Treatment with T, but not DHT or placebo, improved target quadrant bias on the third and final probe trial, indicating a significant improvement in spatial memory. In addition, since there was no effect of either T or DHT in the visible training portion of the water maze task, it is unlikely that performance differences in the water maze are due to motor effects of androgens. Similar results have been recently reported in aged male rats using a "working-memory"-radial-arm water maze task (Bimonte-Nelson et al. 2003). In addition to reducing the number of errors in a trial, $\mathrm{T}$, but not DHT, increased message levels of brain-derived neurotrophic factor in the hippocampus (Bimonte-Nelson et al. 2003). This was interpreted to mean that conversion of $\mathrm{T}$ to $17 \beta$-estradiol was the likely pathway for Ts effects on brain and behavior. This idea is supported by a large literature indicating that $17 \beta$-estradiol can improve spatial memory task performance (Galea et al. 2008). Since T, but not DHT, improved spatial memory in our study, it is likely that the beneficial effects of $\mathrm{T}$ on spatial memory in aged mice are due to aromatization to estradiol. Other investigators have divided aged animals into groups of "learners" and "nonlearners" according to their proximity to the hidden platform during water maze training and have shown that approximately half of aged rats are impaired while the other half perform like younger animals (Smith et al. 2000). Here, we also divided animals into learners and nonlearners according to whether they showed a higher percentage of time swimming in the target quadrant during the probe trial (data not shown). Similar to previous reports (Smith et al. 2000), four of the eight placebo-treated female mice were nonlearners and seven of the 10 DHT-treated mice were nonlearners according to this definition. Interestingly, only four of $11 \mathrm{~T}$-treated mice were nonlearners. Although the number of mice in learner and nonlearner categories in each treatment group were not significantly different by $\chi^{2}$ test (data not shown), this further supports that $\mathrm{T}$, and not DHT, is effective in enhancing spatial memory in aged female mice. In addition, further analysis suggested that the division of water maze performance into learners and nonlearners did not carry over into PA or novel object/novel location memory, suggesting that aging differentially affects performance on these tasks at the level of the individual subject. Also, there was no indication that T or DHT levels were different between water-maze learners and nonlearners.

In contrast to its effects on spatial reference memory, supplementation with DHT greatly improved PA memory performance. Testosterone supplementation also resulted in modestly improved performance over placebo. However, the effect of $\mathrm{T}$ supplementation on PA should be interpreted with caution, as the variation in performance was large. It is possible that a larger sample might reveal a more robust performance improvement. Nonetheless, the present results are in agreement with previous data suggesting that systemic administration of DHT can, under 

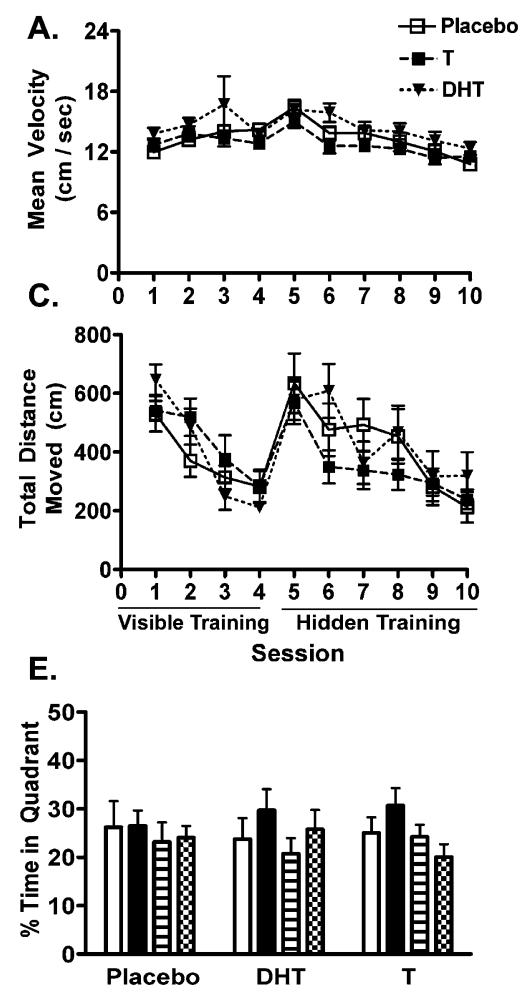

Figure 4. Average swim velocity $(A)$ and learning during the hidden and visible trials of referencememory water maze training as measured by escape latency $(B)$ and total distance moved $(C)$. Also shown are memory retention during the first $(D)$, second $(E)$, and third and final probe trials $(F)$ of the reference-memory Morris water maze. Mice in all groups showed significant performance improvement over the training sessions during both visible and hidden training according to all measures. However, performance was unaffected by either T or DHT supplementation. In contrast, mice that received T supplementation achieved significant spatial bias in the third probe trial, whereas placeboand DHT-treated mice did not. No significant preference for the target quadrant of the maze was observed during either the first or second probe trials for any group. ${ }^{*} P<0.05$, a priori planned contrast.

some circumstances, improve PA memory in rats (Frye and Lacey 2001; Frye et al. 2004). Also, in adult male rats, systemic DHT treatment reverses castration-induced impairment in PA memory (Frye and Seliga 2001). However, ours is the first study to show that DHT can improve cognitive performance in aged female mice. Other studies using hippocampal administration of an inhibitor of DHT metabolism suggest that the beneficial effects of DHT in male rats may be due to the hippocampal actions of its neuro-active metabolite $3 \alpha$-diol (Frye et al. 2004). The beneficial effect of $3 \alpha$ diol is also attributable, at least in part, to post-training memory consolidation effects. Conversion to $3 \alpha$-diol may also mediate the beneficial effects of DHT on PA memory in aged female mice. However, we cannot rule out potential mediation by direct genomic or nongenomic actions of the AR. For example, AR activation has been linked to dendritic spine-synapse density (Leranth et al. 2004), adult hippocampal neurogenesis (Spritzer and Galea 2007), and central neurotransmitter systems, including glutamate (Pouliot et al. 1996; Foradori et al. 2007), dopamine (Kritzer et al. 2007), acetylcholine (Nakamura et al. 2002), and corticotrophinreleasing factor (Gomez et al. 2004). It is interesting that the circulating DHT levels were much higher in the T-treated group compared with the DHT-treated group. Nonetheless, T was only partially effective in improving PA memory performance. There are some possible explanations for this. First, it is possible that estrogen-related effects might counteract androgen-related improvement in PA performance. Alternatively, the level of conversion of $\mathrm{T}$ to DHT in brain might be less than in the periphery, leading to lower levels of DHT in brain regardless of high DHT in plasma. Finally, this could be a dosedependent effect in that very high levels of circulating androgens are less beneficial for PA performance compared with lower levels, as has been suggested for verbal and spatial memory performance in older men (Cherrier et al. 2007). Further study is needed to elucidate the precise reason why $\mathrm{T}$ is only partially effective in enhancing PA performance in aged female mice.

Neither T nor DHT supplementation affected performance on measures of $\mathrm{NO} / \mathrm{NL}$ recognition memory. Within the limits of our study, these results indicate that behavioral performance on this task is androgen insensitive in aged female mice. Whereas age-related disruptions in water maze reference memory and PA memory performance are greater in female mice compared with male mice, age-related decline in $\mathrm{NO} / \mathrm{NL}$ recognition is unaffected by sex (Benice et al. 2006). Although not directly related to androgen levels, these results support the idea that age-related decline in $\mathrm{NO} / \mathrm{NL}$ recognition may be unaffected by reduced androgen levels. Also, NL recognition declines with age in mice but NO recognition does not (Benice et al. 2006). Thus, an androgen-induced performance enhancement in NO performance would not necessarily be expected. In addition, in adult male testicular feminization mutation (TFM) mice, which have impaired AR signaling, both NO and NL recognition is intact (Rizk et al. 2005). However, adult female ovariectomized rats show improvements in NO performance when given T or DHT immediately following training, indicating that androgen supplementation can improve NO performance in some circumstances (Frye and Lacey 2001). However, the NO task in that study was different from the one used in the current study. In that study, rats were tested $24 \mathrm{~h}$ after training, whereas here, mice were tested $10 \mathrm{~min}$ following training. It has been shown that NO performance becomes hippocampus dependent with a 24-h delay between training and testing but not with a 10-min delay (Hammond et al. 2004). Thus, different brain areas were likely involved in performance between that study and the present study. In addition,

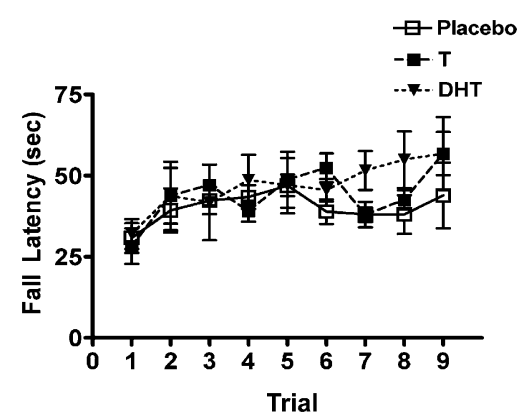

Figure 5. Roto-rod performance. Although there was significant performance improvement over the nine training trails for all groups, there was no effect of either T or DHT supplementation. 
A.

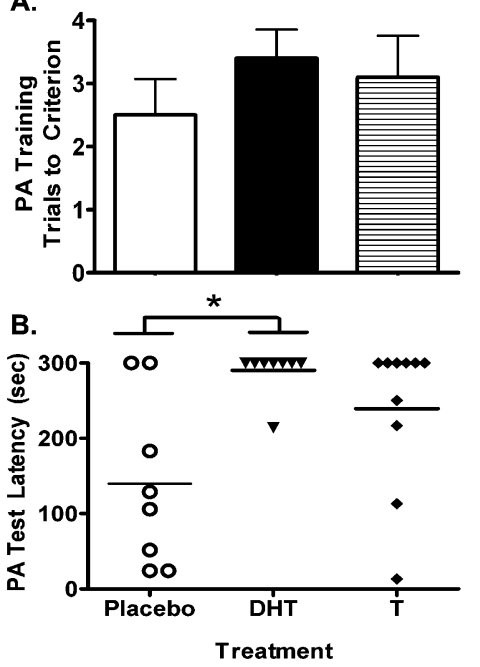

Figure 6. Passive avoidance (PA) training performance $(A)$ and memory performance $(B), 24 \mathrm{~h}$ following training. Neither T nor DHT supplementation affected training performance as measured by the number of trials to criterion (A). In contrast, compared with either T- or placebo-treated mice, mice that received DHT supplementation showed significantly elevated latencies to enter the dark chamber (PA test latency) $24 \mathrm{~h}$ after training, indicating improved passive avoidance memory $(B)$. In contrast, performance was not improved in T-treated mice compared with placebo-treated mice. Each data point in the figure $(B)$ represents the actual PA test latency for each mouse. ${ }^{*} P<$ 0.05 by Mann-Whitney $U$-test following Kruskall-Wallis test.

age-related impairments in NO performance are exacerbated by ovariectomy in aged female mice (Vaucher et al. 2002) and are improved by estradiol treatments in middle-aged mice (Gresack et al. 2007). Thus, it is interesting that $T$ treatment did not enhance $\mathrm{NO} / \mathrm{NL}$ performance in our study as $\mathrm{T}$ is readily aromatized to estradiol. However, the levels of aromatization in the brain are unknown in aged female mice; thus, there could have been a lack of such aromatization. Alternatively, AR stimulation by $\mathrm{T}$ might have somehow counteracted estradiol-related performance enhancements. It is important to note that estradiol treatment, at least when given after training, enhanced performance in middle-aged, but not aged, female mice (Gresack et al. 2007). This is consistent with our data in suggesting that aromatization of $\mathrm{T}$ to estradiol may be ineffective in enhancing $\mathrm{NO} / \mathrm{NL}$ performance in old female mice.

Androgen supplementation did not affect anxiety in the open field test. Androgens are linked to stress responsiveness and anxiety-like behavior through their actions on corticotrophinreleasing hormone and arginine-vasopressin neurons in the hypothalamus and amygdala (McCormick et al. 2002; Gomez et al. 2004). In general, androgens are considered anxiolytic, due in part to the effects of the $3 \alpha$-reduced neurosteroid $3 \alpha$-diol on GABA-A receptors (Frye and Seliga 2001; Patchev et al. 2004). However, our data suggest that the anxiolytic effects of androgens, which have been shown exclusively in adult animals, may not be potent in aged animals. Furthermore, there is little data concerning androgeninduced anxiolysis in female rodents. Our data suggest that sex differences should be examined closely in future studies of mood elevation using androgen-based therapies.

The differential response of the cognitive tasks to T and DHT treatment begs the question whether these hormones differentially affect the neural circuits underlying spatial reference memory, object recognition memory, and PA memory. It is well known that hippocampal damage impairs spatial reference memory (Morris et al. 1982) and PA memory (Stubley-Weatherly et al. 1996), but not object recognition memory with delays less than 24-h between learning and testing (Hammond et al. 2004). In addition, lesions of the amygdala impair PA memory (Swartzwelder 1981) while leaving spatial reference memory intact (Spanis et al. 1999). Finally, lesions of the parahippocampal regions including the perirhinal cortex impair object recognition memory (Ennaceur and Aggleton 1997). Thus, it is interesting to speculate that in aged female mice, $\mathrm{T}$ mainly improves spatial reference memory by acting on the hippocampus, DHT improves PA memory by acting on the amygdala, and neither hormone affects the function of parahippocampal brain regions. Future studies must specifically target these brain regions with hormone treatments to add evidence to this interesting speculation.

In conclusion, in aged female mice, hormone supplementation with T improved spatial reference memory and DHT improved PA memory. Neither treatment affected anxiety in the open field. These data support the hypothesis that androgenic mechanisms can benefit cognition in elderly female rodents, especially in tasks such as PA in which the hippocampus and amygdala are implicated. Future studies are warranted to determine the molecular mechanisms underlying these differential therapeutic effects.

\section{Materials and Methods}

\section{Mice}

Female C57BL/6J mice were bred in our colony and housed three to five per cage until they were $20-22$ mo of age, at which point surgery took place (see procedure below). Behavioral testing commenced 6 wk following surgery. The mice remained gonadally intact during hormone supplementation and behavioral testing to be consistent with our previous study of ACD in C57BL/6J mice (Benice et al. 2006). All mice were reproductively naïve. We (Benice et al. 2006), and others (Nelson et al. 1982), have found in previous research that female $\mathrm{C} 57 \mathrm{BL} / 6 \mathrm{~J}$ mice aged older than 18 mo are in permanent diestrous, indicating negligible levels of circulating sex steroids. Food (PicoLab Rodent Diet 20, no. 5053; PMI Nutrition International) and water were provided ad libitum, and there was a constant 12-on/12-off light cycle (on 6:00 a.m., off 6:00 p.m.). Mice were singly housed starting $2 \mathrm{~d}$ before the first behavioral test to eliminate potential social effects (including hierarchy in the home cage and stress of removing a mouse from the cage on remaining mice in the cage) on behavioral performance. Behavioral testing lasted $4 \mathrm{wk}$. Within $48 \mathrm{~h}$ of the end of the last behavioral test, blood samples were taken to measure plasma hormone levels (see procedure below).

\section{Hormone supplementation and measurement of hormone levels}

The mice were $20-22$ mo of age at the time of surgery. Each mouse was deeply anesthetized using isofluorane in $\mathrm{O}_{2}$, and a $0.5-\mathrm{cm}$ incision was made in the skin between the shoulder blades. A silastic capsule (inner diameter $=1.57 \mathrm{~mm}$; outer diameter $=3.18$ $\mathrm{mm}$; length $=2.5 \mathrm{~cm})$ containing either T or DHT $($ Sigma) or remaining empty (placebo) was implanted subcutaneously and the wound sutured. To measure the effectiveness of the capsules in increasing circulating hormone levels, plasma samples were taken following behavioral testing in a subsample of mice from each group (placebo, $n=5$; T, $n=6$; DHT, $n=6$ ) and were subjected to ELISA analysis for concentrations of $\mathrm{T}$ and DHT using commercially available kits (Alpha Diagnostic International) according to the supplier's instructions. For plasma samples, blood was taken from the heart into Eppendorf tubes containing $\sim 10 \mu \mathrm{L}$ of a $5 \%$ EDTA solution. The blood was centrifuged for $10 \mathrm{~min}$ at $16,000 \mathrm{~g}$. The plasma was then separated and stored at $-20^{\circ} \mathrm{C}$ until assay.

\section{Behavioral testing}

The behavioral tasks were done in the order presented below. The entire testing battery lasted 4 wk with 1-2 d elapsing between tasks. All procedures followed those used in a previous study (Benice et al. 2006) and are described briefly below. 


\section{Open field test}

Open field activity was assessed individually for $10 \mathrm{~min}$ in brightly lit enclosures (16-in $\times 16$-in square) equipped with a $16 \times 16$ array of infrared photocells for measuring horizontal movements, and quantified automatically by computer (Kinder Scientific). Total distance moved measured activity levels and percentage of time spent in the center of the enclosure was used as a measure of anxiety-like behavior (Clement et al. 2002). After each assessment of open field activity, the equipment was cleaned with $5 \%$ acetic acid to remove residual odors.

\section{NO/NL recognition}

For three consecutive days, the mice were individually habituated to a 16-in $\times 16$-in square-shaped open field with clear Plexiglas walls for $5 \mathrm{~min}$ (Hamilton-Kinder). On the fourth day, the mice were first trained in three consecutive 10-min familiarization trials and then were tested in two consecutive 10-min trials, each with a 5 -min intertrial interval. For the familiarization trials, three plastic toy objects were placed in the open field (one in each of three corners), and an individual animal was allowed to explore for $10 \mathrm{~min}$. All familiar objects were exchanged with replicas in subsequent trials. After three familiarization trials, the mouse was tested in a location novelty recognition test in which one of the familiar objects was moved to a novel location in the arena. The same object was moved to the same new location for every mouse tested. The mouse was then tested in an object novelty recognition test in which a novel object replaced one of the familiar objects. All objects and the arena were thoroughly cleaned with $5 \%$ acetic acid between trials to remove odors.

The time spent exploring each object during the familiarization training and the testing trials was recorded by a trained observer. Exploration was defined as approaching the object nose first within $2-4 \mathrm{~cm}$. The time spent exploring each object, as a percentage of the total exploration time, was calculated for each trial. The difference between the percentage of time spent exploring the object in the novel location (trial 4) and the percentage of time spent exploring the same object in its original location on the previous trial (trial 3) was calculated to measure novel location recognition. The percentage of time spent exploring the novel object during trial 5 minus the percentage of time spent exploring the familiar object in the same location on trial 4 was calculated to measure novel object recognition. To be sure that motivation to explore the objects was similar between the groups, we also analyzed the total time exploring the objects over the five trials.

\section{Reference-memory water maze}

\section{Training}

A circular pool (diameter, $140 \mathrm{~cm}$ ) was filled with opaque water $\left(24^{\circ} \mathrm{C}\right)$, and mice were trained to swim to a submerged platform in order to escape from the water. First they were trained with the platform clearly marked with a beacon during the visible platform component (nonspatial training, days 1 and 2, sessions 1-4). Mice were then trained with the beacon removed in the hidden platform component (spatial training, days 3-5, sessions 5-10), during which the mice had to navigate by using the available spatial cues in the room (posters on the walls and assorted apparatus on benches). Whereas the visible component of training is not sensitive to hippocampal lesions, the hidden component of training is (Morris et al. 1982). In order to avoid any quadrant bias, mice were assigned to four blocking groups using a randomized block design with the starting quadrant as the blocking factor, and each blocking group started training in a different quadrant of the pool. Thenceforth, during the visible platform training, the platform was moved to a different quadrant of the pool for each session. In contrast, for the hidden platform training, the platform location was kept constant for each blocking group with the hidden platform location being the same as the location in the first visible platform session.

There were two daily sessions $3.5 \mathrm{~h}$ apart, each consisting of three 60-sec trials (with 10- to 15-min intertrial intervals). Mice that failed to find the platform within $60 \mathrm{sec}$ were led by the experimenter to the platform, where they were allowed to stay for $3 \mathrm{sec}$. Mice were placed into the water facing the wall at the side of the pool in nine different locations around the pool circumference, and the starting location was changed for each trial. The swimming patterns of the mice were recorded at six samples/sec with a Noldus Instrument Ethovision video tracking system (Noldus Information Technology). To measure performance in both the visible and hidden training, the latency to find the platform (escape latency, seconds), total distance moved (centimeters), and average swim speed were analyzed as outcome measures for each session.

\section{Probe trial testing}

The probe trials were designed to examine the extent of spatial discrimination learning (spatial bias) at three different time points during hidden platform training. To do this, the platform was removed from the pool $\sim 1 \mathrm{~h}$ after the last hidden platform training trial of each day of hidden training (total of three probe trials), and the time that mice spent swimming in the target quadrant (where the platform was located during hidden platform training), and in the three nontarget quadrants (right, left, and opposite quadrant) was measured over a 60 -sec trial. Preferential searching was indicated by more time spent in the target quadrant than any nontarget quadrant.

\section{Roto-rod}

Roto-rod was used to test potential effects of hormone supplementation on a variety of proprioceptive, vestibular, and finetuned motor abilities. In this task, mice balanced on a $7-\mathrm{cm}$ diameter rotating rod (Kinder Scientific). After a 1-min adaptation period without rotation, the rod was accelerated by 5 rpm every 15 sec, and the latency to fall from the rod was recorded. Each mouse received $3 \mathrm{~d}$ of roto-rod testing with three trials per day and a $\sim 20$ min intertrial interval. The average fall latency for all trials was used as the measure of motor coordination.

\section{Passive avoidance (PA)}

PA performance was measured with a step-through box consisting of a brightly lit compartment and an identical dark compartment connected with a sliding door (Hamilton-Kinder). Mice were placed individually in the bright compartment, and after a habituation period of $5 \mathrm{sec}$, the door was opened into the darkened compartment. Mice, being averse to bright light, were naturally inclined to enter the darkened compartment. When they did so, the door quickly shut and a slight footshock was delivered ( $0.3 \mathrm{~mA}$ for $3 \mathrm{sec})$. Each mouse was trained until it met a learning criterion of three consecutive 120 -sec trials without entering the darkened compartment or it completed to 10 trials, whichever came first. Twenty-four hours later, each mouse was once again placed into the bright compartment, and the latency to re-enter the dark compartment was recorded up to $300 \mathrm{sec}$. The number of trials to criterion was used to measure PA acquisition, and time before entering the dark chamber $24 \mathrm{~h}$ after training was used to measure PA memory.

\section{Statistical analysis}

For plasma hormone levels, open field test, and the NO/NL tasks, the effect of hormone treatment as a between-subjects factor was measured by one-way fixed ANOVA. Reference-memory water maze training performance and total time exploring the objects in the NO/NL trials were analyzed using two-way mixed ANOVA with hormone treatment as the between-subjects factor and training session as the within-subjects factor. For the water-maze probe trials, we expected a priori to see higher preference for the target quadrant over the other four quadrants. Thus, to measure whether there was spatial bias for the target quadrant in each probe trial for each group, a priori contrasts were used comparing time spent swimming in the target quadrant vs. all other quadrants. The contrast coefficients used were as follows: percent time swimming in the target quadrant $=$ 1 , percent time swimming in the left quadrant $=-1 / 3$, percent time swimming in the right quadrant $=-1 / 3$, and percent time swimming in the opposite quadrant $=-1 / 3$. Roto-rod performance was analyzed in the same fashion as water maze training performance, except trial number was the within-subjects factor. The effects of hormone 
treatment on PA acquisition and memory performance were analyzed using Kruskal-Wallis nonparametric test. For the ANOVA analyses, Duncan's post-hoc test was used to measure differences between individual groups where appropriate. Kruskal-Wallis tests were followed by Mann-Whitney $U$-tests to compare individual groups.

\section{Acknowledgments}

This work was supported by 5T32AG023477-02, T32 NS007466-05, NASA NNJ05HE63G, and Alzheimer's Association IIRG-05-14021.

\section{References}

Benice TS, Rizk A, Kohama S, Pfankuch T, Raber J. 2006. Sex-differences in age-related cognitive decline in C57BL/6J mice associated with increased brain microtubule-associated protein 2 and synaptophysin immunoreactivity. Neuroscience 137: 413-423.

Bimonte-Nelson HA, Singleton RS, Nelson ME, Eckman CB, Barber J, Scott TY, Granholm AC. 2003. Testosterone, but not nonaromatizable dihydrotestosterone, improves working memory and alters nerve growth factor levels in aged male rats. Exp Neurol 181: 301-312.

Cherrier MM, Matsumoto AM, Amory JK, Johnson M, Craft S, Peskind ER, Raskind MA. 2007. Characterization of verbal and spatial memory changes from moderate to supraphysiological increases in serum testosterone in healthy older men. Psychoneuroendocrinology 32: 72-79.

Clement Y, Calatayud F, Belzung C. 2002. Genetic basis of anxiety-like behaviour: A critical review. Brain Res Bull 57: 57-71.

Cooke BM. 2006. Steroid-dependent plasticity in the medial amygdala. Neuroscience 138: 997-1005.

Driscoll I, Hamilton DA, Petropoulos H, Yeo RA, Brooks WM, Baumgartner RN, Sutherland RJ. 2003. The aging hippocampus: Cognitive, biochemical and structural findings. Cereb Cortex 13: 1344-1351.

Driscoll I, Hamilton DA, Yeo RA, Brooks WM, Sutherland RJ. 2005. Virtual navigation in humans: The impact of age, sex, and hormones on place learning. Horm Behav 47: 326-335.

Ennaceur A, Aggleton JP. 1997. The effects of neurotoxic lesions of the perirhinal cortex combined to fornix transection on object recognition memory in the rat. Behav Brain Res 88: 181-193.

Foradori CD, Werner SB, Sandau US, Clapp TR, Handa RJ. 2007. Activation of the androgen receptor alters the intracellular calcium response to glutamate in primary hippocampal neurons and modulates sarco/ endoplasmic reticulum calcium ATPase 2 transcription. Neuroscience 149: $155-164$

Frick KM, Burlingame LA, Arters JA, Berger-Sweeney J. 2000. Reference memory, anxiety and estrous cyclicity in C57BL/6NIA mice are affected by age and sex. Neuroscience 95: 293-307.

Frick KM, Fernandez SM, Bulinski SC. 2002. Estrogen replacement improves spatial reference memory and increases hippocampal synaptophysin in aged female mice. Neuroscience 115: 547-558.

Frye CA, Lacey EH. 2001. Posttraining androgens' enhancement of cognitive performance is temporally distinct from androgens' increases in affective behavior. Cogn Affect Behav Neurosci 1: 172-182.

Frye CA, Seliga AM. 2001. Testosterone increases analgesia, anxiolysis, and cognitive performance of male rats. Cogn Affect Behav Neurosci 1: 371-381.

Frye CA, Edinger KL, Seliga AM, Wawrzycki JM. 2004. 5 $\alpha$-Reduced androgens may have actions in the hippocampus to enhance cognitive performance of male rats. Psychoneuroendocrinology 29: 1019-1027.

Frye CA, Rhodes ME, Dudek B. 2005. Estradiol to aged female or male mice improves learning in inhibitory avoidance and water maze tasks. Brain Res 1036: 101-108.

Galea LA, Uban KA, Epp JR, Brummelte S, Barha CK, Wilson WL, Lieblich SE, Pawluski JL. 2008. Endocrine regulation of cognition and neuroplasticity: Our pursuit to unveil the complex interaction between hormones, the brain, and behaviour. Can J Exp Psychol 62: 247-260.

Gomez F, Manalo S, Dallman MF, Gomez F, Manalo S, Dallman MF. 2004. Androgen-sensitive changes in regulation of restraint-induced adrenocorticotropin secretion between early and late puberty in male rats. Endocrinology 145: 59-70.

Gresack JE, Kerr KM, Frick KM. 2007. Life-long environmental enrichment differentially affects the mnemonic response to estrogen in young, middle-aged, and aged female mice. Neurobiol Learn Mem 88: 393-408.

Hammond RS, Tull LE, Stackman RW. 2004. On the delay-dependent involvement of the hippocampus in object recognition memory. Neurobiol Learn Mem 82: 26-34.

Hanninen T, Hallikainen M, Koivisto K, Partanen K, Laakso MP, Riekkinen PJ Sr, Soininen H. 1997. Decline of frontal lobe functions in subjects with age-associated memory impairment. Neurology 48: 148-153.

Isgor C, Sengelaub DR. 1998. Prenatal gonadal steroids affect adult spatial behavior, CA1 and CA3 pyramidal cell morphology in rats. Horm Behav 34: $183-198$.
Kritzer MF, Brewer A, Montalmant F, Davenport M, Robinson JK. 2007. Effects of gonadectomy on performance in operant tasks measuring prefrontal cortical function in adult male rats. Horm Behav 51: 183-194.

Leranth C, Hajszan T, MacLusky NJ. 2004. Androgens increase spine synapse density in the CA1 hippocampal subfield of ovariectomized female rats. J Neurosci 24: 495-499.

Lu S-F, McKenna SE, Cologer-Clifford A, Nau EA, Simon NG. 1998. Androgen receptor in mouse brain: Sex differences and similarities in autoregulation. Endocrinology 139: 1594-1601.

Markowska AL. 1999. Sex dimorphisms in the rate of age-related decline in spatial memory: Relevance to alterations in the estrous cycle. J Neurosci 19: 8122-8133.

Markowska AL, Savonenko AV. 2002. Effectiveness of estrogen replacement in restoration of cognitive function after long-term estrogen withdrawal in aging rats. J Neurosci 22: 10985-10995.

McCormick CM, Linkroum W, Sallinen BJ, Miller NW. 2002. Peripheral and central sex steroids have differential effects on the HPA axis of male and female rats. Stress 5: 235-247.

McPhaul M, Young M. 2001. Complexities of androgen action. J Am Acad Dermatol 45: S87-S94.

Morris RG, Garrud P, Rawlins JN, O'Keefe J. 1982. Place navigation impaired in rats with hippocampal lesions. Nature 297: 681-683.

Morris JA, Jordan CL, Breedlove SM. 2008. Sexual dimorphism in neuronal number of the posterodorsal medial amygdala is independent of circulating androgens and regional volume in adult rats. J Comp Neurol 506: $851-859$.

Nakamura N, Fujita H, Kawata M. 2002. Effects of gonadectomy on immunoreactivity for choline acetyltransferase in the cortex, hippocampus, and basal forebrain of adult male rats. Neuroscience 109: 473-485.

Nelson JF, Felicio LS, Randall PK, Sims C, Finch CE. 1982. A longitudinal study of estrous cyclicity in aging C57BL/6J mice: I. Cycle frequency, length and vaginal cytology. Biol Reprod 27: 327-339.

Patchev VK, Schroeder J, Goetz F, Rohde W, Patchev AV. 2004. Neurotropic action of androgens: Principles, mechanisms and novel targets. Exp Gerontol 39: 1651-1660.

Pouliot WA, Handa RJ, Beck SG. 1996. Androgen modulates N-methyl-Daspartate-mediated depolarization in CA1 hippocampal pyramidal cells. Synapse 23: 10-19.

Raber J, Wong D, Yu GQ, Buttini M, Mahley RW, Pitas RE, Mucke L. 2000. Apolipoprotein E and cognitive performance. Nature 404: 352-354.

Raber J, Bongers G, LeFevour A, Buttini M, Mucke L. 2002. Androgens protect against apolipoprotein E4-induced cognitive deficits. J Neurosci 22: 5204-5209.

Rizk A, Robertson J, Raber J. 2005. Behavioral performance of $t f m$ mice supports the beneficial role of androgen receptors in spatial learning and memory. Brain Res 1034: 132-138.

Silbert LC, Nelson C, Howieson DB, Moore MM, Kaye JA. 2008. Impact of white matter hyperintensity volume progression on rate of cognitive and motor decline. Neurology 71: 108-113.

Simerly RB, Chang C, Muramatsu M, Swanson LW. 1990. Distribution of androgen and estrogen receptor mRNA-containing cells in the rat brain: An in situ hybridization study. I Comp Neurol 294: 76-95.

Small GW, La Rue A, Komo S, Kaplan A, Mandelkern MA. 1995. Predictors of cognitive change in middle-aged and older adults with memory loss. Am J Psychiatry 152: 1757-1764.

Smith TD, Adams MM, Gallagher M, Morrison JH, Rapp PR. 2000. Circuitspecific alterations in hippocampal synaptophysin immunoreactivity predict spatial learning impairment in aged rats. J Neurosci 20: 6587-6593.

Smith CD, Chebrolu H, Wekstein DR, Schmitt FA, Markesbery WR. 2007. Age and gender effects on human brain anatomy: A voxel-based morphometric study in healthy elderly. Neurobiol Aging 28: 1075-1087.

Spanis CW, Bianchin MM, Izquierdo I, McGaugh JL. 1999. Excitotoxic basolateral amygdala lesions potentiate the memory impairment effect of muscimol injected into the medial septal area. Brain Res 816: 329-336.

Spritzer MD, Galea LAM. 2007. Testosterone and dihydrotestosterone, but not estradiol, enhance survival of new hippocampal neurons in adult male rats. Dev Neurobiol 67: 1321-1333.

Stubley-Weatherly L, Harding JW, Wright JW. 1996. Effects of discrete kainic acid-induced hippocampal lesions on spatial and contextual learning and memory in rats. Brain Res 716: 29-38.

Swartzwelder HS. 1981. Deficits in passive avoidance and fear behavior following bilateral and unilateral amygdala lesions in mice. Physiol Behav 26: 323-326.

Tisserand DJ, Jolles J. 2003. On the involvement of prefrontal networks in cognitive ageing. Cortex 39: 1107-1128.

Vaucher E, Reymond I, Najaffe R, Kar S, Quirion R, Miller MM, Franklin KB. 2002. Estrogen effects on object memory and cholinergic receptors in young and old female mice. Neurobiol Aging 23: 87-95.

Received March 20, 2009; accepted in revised form June 1, 2009. 


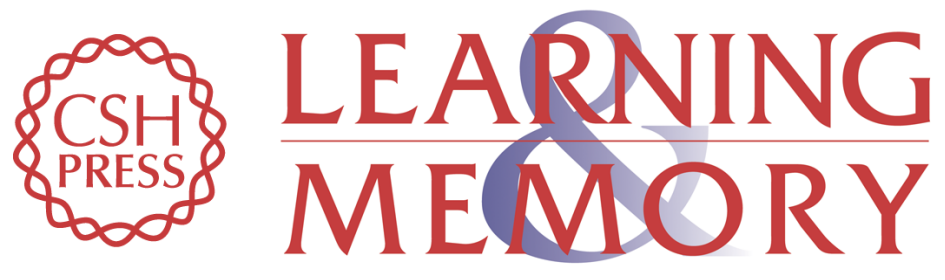

\section{Testosterone and dihydrotestosterone differentially improve cognition in aged female mice}

Ted S. Benice and Jacob Raber

Learn. Mem. 2009, 16:

Access the most recent version at doi:10.1101//m.1428209

References This article cites 48 articles, 7 of which can be accessed free at:

http://learnmem.cshlp.org/content/16/8/479.full.html\#ref-list-1

License

Email Alerting Receive free email alerts when new articles cite this article - sign up in the box at the Service top right corner of the article or click here. 